\title{
Integrated experimental and computational pipeline for proteome- wide in tissue crosslinking analysis
}

\author{
K.A.T. Makepeace ${ }^{1}$, Y. Mohammed ${ }^{1,2}$, E.L. Rudashevskaya ${ }^{3}$, R.D. Brown', \\ E.V. Petrotchenko ${ }^{1 *}$, A. Sickmann ${ }^{3}$, C.H. Borchers ${ }^{1,4}$ \\ ${ }^{1}$ University of Victoria - Genome British Columbia Protein Centre, University of Victoria, \\ Vancouver Island Technology Park, Victoria, Canada \\ ${ }^{2}$ Center for Proteomics and Metabolomics, Leiden University, Leiden, Netherlands \\ ${ }^{3}$ Leibniz Institut für Analytische Wissenschaften - e.V., Dortmund, Germany \\ ${ }^{4}$ Dept of Biochemistry and Microbiology, University of Victoria, Victoria, Canada \\ *e-mail: jenyavp@gmail.com
}

Key words: proteome, protein structure, crosslinking analysis, peptides

Proteome-wide crosslinking analysis has the potential for the structural characterization of proteins in their natural cellular and tissue environments. The technique is able to capture weakly interacting and transient complexes, and can provide structural information on protein-protein interaction interfaces and conformations. The analysis is performed by chemical crosslinking proteins in situ, enzymatically digesting the crosslinked proteins, and identifying the crosslinked peptides using MS. Although the process is fairly straightforward, for proteome-wide applications the approach is dramatically complicated by the combinatorial nature of crosslinked peptides, requiring the identification of the interacting proteins based on a single peptide from each protein in a crosslinked peptide pair. Here, we describe the complete experimental and computational workflow using the isotopically-coded affinity-enrichable CID-cleavable crosslinker CBDPS. 